\title{
Mesoscale investigations for the evolution of interfaces in plasticity
}

\author{
Irene J. Beyerlein ${ }^{1}$ and Jason R. Mayeur
}

Theoretical Division, Los Alamos National Laboratory, Los Alamos, NM 87545 USA

\begin{abstract}
:
The ultra-high density of bimetal interfaces in nanolayered metal composites is responsible for exceptional five- to ten-fold increases in strength over that of the constituent phases. How interfaces cause such outstanding results is not fully understood. On one hand, interfaces fundamentally alter the physical mechanisms responsible for plastic deformation-an important facet of the problem that has been intensely studied. On the other hand, plastic deformation also results in the creation of new interfaces. While this second facet holds equal importance, it has received comparatively less attention. Further, these two facets, coined interfacedriven plasticity and plasticity-driven interfaces, are naturally coupled processes during deformation. The time and length scales characteristic of mesoscale characterization and simulation methods make them well suited for understanding their connection. Along these lines, here we focus on the use of mesoscale characterization and modeling to explore the role that bimetal interfaces play in their own self-development during severe plastic deformation. We demonstrate how mesoscale modeling can provide valuable insight into the design of materials processing techniques with stable interfaces in mind.
\end{abstract}

Keywords: dislocations, crystal plasticity, interfaces, stability, severe plastic deformation

\section{Highlights:}

- Nanostructuring in two-phase metals can be achieved by severe plastic deformation (SPD)

- SPD can be used to create specific bimetal interfaces

- Plasticity-driven interfaces and interface-driven plasticity are interrelated

- Mesoscales, not atomic scales, capture interface effects on texture

- Mesoscale models are introduced that predict interface-driven texture evolution

\subsection{Introduction}

In the design of next generation structural metals, the principal aim is to increase strength several fold [1,2]. This includes yield and ultimate strengths or more generally, the stress required to initiate plastic flow at a given strain. These

${ }^{1}$ Corresponding author: Irene@lanl.gov, 505-665-2231 
properties are extremely sensitive to the underlying plastic deformation mechanisms and the evolving microstructure at microscopic scales and lower.

Materials that can potentially meet such demands are two-phase (bimetal) nanolayered metals. In recent years, studies have reported ultra-high strength and robustness in extreme environments [3-8]. Nanolayered metals exhibit strengths that are five to ten times stronger $[7,9,10]$ and hardnesses that are two to three orders of magnitude higher $[9,11,12]$ than those of their constituents. Moreover, the strengths and hardness are far more stable at elevated temperatures than their constituents $[13,14]$. These outstanding qualities cannot be explained by a simple volume average of their constituent properties [7].

The exceptional plastic properties of nanolayered composites have been attributed to their bimetal interfaces, the boundary between two crystals of dissimilar metals. Over a broad range of bimetal interface densities, nanolayered composite properties are found to be strong functions of the individual layer thickness, or the spacing between adjacent interfaces $h$, often improving as $h$ decreases $[7,15,16]$. It is commonly believed that when $h$ shrinks to submicron and nanoscale dimensions, the interfaces dominate the plastic response and either alter or lead to unusual plastic deformation mechanisms. However, how interfaces enable such responses has yet to be clarified.

In full recognition of this knowledge gap, many studies have used nanomechanical testing and numerical tools like molecular dynamics (MD), discrete dislocation dynamics, and phase-field based techniques, to study basic dislocation processes in nanocomposites and nanocrystals [17-23]. The motion of dislocations underlies plastic deformation responses and material strength. Thus, a key question is how do interfaces modify the glide of a dislocation(s) and the interactions between an interface and one or a few dislocations? So far, the results have revealed that interface structure can profoundly affect dislocation nucleation [21, 24, 25], dislocation transmission [26-28], dislocation recovery (annihilation) [17], and twinning dislocation nucleation [29-31]. From these small-scale studies, it is suggested that interfaces can drive plasticity. This lies in significant contrast to conventional metals, in which dislocation processes occurring within the crystals are thought to drive plastic deformation.

Interface-driven plasticity is only one facet of the problem in understanding the plastic behavior of nanostructured metals. The reciprocal operation, plasticitydriven interface evolution, is another important facet. While a metal is plastically deforming, the microstructure is not stationary but can evolve substantially [32-34]. The crystals can deform into different shapes, they can reorient crystallographically, the grain boundaries can change character, and dislocations can be generated and stored. Most significantly new interfaces can be created. This is the essence of grain refinement, a process that is used by severe plastic deformation (SPD) techniques to reduce grain sizes from $\mathrm{mm}$ to submicrometer and nanometer dimensions. These additionally generated interfaces are plastically driven. 
Plasticity-driven interfaces and interface-driven plasticity are related. The former, which is of equal importance as the latter, has not received as much attention. Mesoscale characterization and modeling have the potential to study the interrelationship between them. The time and length scales within the meso-regime are well suited to address how interfaces affect crystal plasticity and in turn how crystal plasticity affects the types of interfaces that are created.

As a platform to draw attention to this need, in this article, we study the creation of stable bimetal interfaces in "bulk" nanolayered composites during plastic deformation. We focus on how mesoscale characterization and modeling were vital to understanding how plasticity affects the properties of newly formed interfaces. The findings show that interfaces can have a significant influence on microstructural evolution in nanomaterials. They also expose more refined questions that can potentially be addressed by mesoscale techniques. The effect of interfaces on the evolution of microstructure, including their own self-creation, during plastic deformation is a wide-open research area. In this frontier, a mesoscale point-ofview has the potential to lead to improved prediction, control, and design of interfaces within bulk nanolayered metal composites for exceptional strength and stability.

\subsection{Plasticity created bimetal interfaces}

As pointed out in the introduction, a paramount example of utilizing plasticity to create internal boundaries in metals is found in the process of severe plastic deformation (SPD). Over the past few decades, SPD techniques have grown into mature metalworking approaches for nanostructuring metals in bulk [34]. These processing methods exploit extreme plastic strains to refine the microstructure, thereby creating new grain boundary and/or interface area that leads to an increase in strength.

Most SPD studies to date focus on single-phase, monolithic metals. However, there are a few studies in which SPD techniques, such as accumulative roll bonding (ARB), accumulative wire drawing and bundling, and equal channel angular extrusion, have also been applied to two-phase metals, such as Ag-Fe, $\mathrm{Cu}-\mathrm{Fe}, \mathrm{Ag}-\mathrm{Ni}, \mathrm{Zr}-\mathrm{Nb}$, and Ag$\mathrm{Cu}$ [35-38]. In monolithic materials, coarse-grain sizes on the order of $\mathrm{mm}$ can be refined to a material-dependent saturation grain size, $D_{\text {sat }}$, which typically lies in the range of several hundreds to tens of nanometers. For two-phase polycrystalline metals, SPD creates bimetal interfaces in addition to grain boundaries (Fig. 1).

Fig. 1 (a) The severe plastic deformation process, accumulative roll bonding (ARB), used to synthesize, (b) $\mathrm{Cu}-\mathrm{Nb}$ nanolayered composites of controllable layer thickness. (c) Transmission electron microcopy (TEM) micrograph displaying the planar $\mathrm{Cu}-\mathrm{Nb}$ interfaces in an $h=20 \mathrm{~nm}$ composite. Taken with permissions from [39]. 
Fig. 1 illustrates the substantial microstructural evolution of a two-phase material during the ARB process. The ARB process involves a sequence of repeated rolling, cutting, and restacking Fig. 1a [35]. At the beginning of the process, the metals are coarse-grained sheets with many crystals spanning the distance between two adjacent phase interfaces. With further straining, the spacing between neighboring bimetal interfaces, $h$, refines; the bimetal interface density increases, and the grains in each phase become elongated in the rolling direction and finer in the thickness dimension (Fig. 2a). The microstructural refinement continues with additional processing until the grain size in both polycrystalline phases reaches their respective saturation grain size $D_{\text {sat }}$ (Fig. $2 \mathrm{~b}$ ). As more ARB steps are added, $h$ is further reduced and bimetal interface area increases, while the average grain size in the polycrystalline layers persists at $D_{\text {sat }}$. Eventually when the layer thickness is refined to $D_{\text {sat }}$ or below, a single crystal spans the layer thickness (Fig. 2c). Reducing the layer thickness beyond this scale results in the creation of more bimetal interface and an increase in the grain dimension in the rolling direction.

Figure 2. Schematic of a predominant interface character that emerges in $\mathrm{Cu}-\mathrm{Nb}$ nanolayered composites after extremely large rolling strains. (a) After straining, the grains become elongated and finer, bimetal interface area is created, and the individual layer thickness becomes finer. Several grains span the individual layers. (b) After more straining, the grains become elongated and reach a saturation size. The bimetal interface area continues to increase and the layers are even finer. (c) After further straining, the layers have refined to thickness that is less than the saturated grain size. One grain spans the layer thickness. The interface area continues to increase and the layers continue to refine.

A recent series of studies considering ARB of a "model" two-phase material system, $\mathrm{Cu}-\mathrm{Nb}$, were carried out to understand the interplay of interfaces and crystal plasticity in microstructural evolution. The starting structure was a multilayer stack of alternating $1 \mathrm{~mm} \mathrm{Cu}$ and $\mathrm{Nb}$ sheets in equal fraction (50/50) [35]. As the plastic strains increased (larger rolling reductions), the individual layer thickness, $h$, decreased uniformly in both $\mathrm{Cu}$ and $\mathrm{Nb}$ and the $\mathrm{Cu}-\mathrm{Nb}$ interface density increased (Fig. 1b) [40]. The newly formed bimetal interfaces were planar and intact for all $h$ down to $20 \mathrm{~nm}$ (Fig. 1c) [19].

\subsection{Preferred bimetal interfaces emerging from extreme straining}

\subsection{Creation of stable interfaces by plasticity}

One way to detect dominance of crystal plasticity is to measure deformation texture. In conventional metal rolling, grains will deform by slip and/or twinning to one of many ideal rolling orientations. These are well-studied and familiar for monolithic metals (without bimetal interfaces) [33, 41]. Ideal rolling components by definition achieve zero reorientation rates in rolling and hence can deform without reorienting. If crystal plasticity dominates, then a distribution of stable rolling components would arise. 
The $\mathrm{Cu}-\mathrm{Nb}$ textures were measured via neutron diffraction for samples, with $h$ from $45 \mu \mathrm{m}$ to $10 \mathrm{~nm}[35,42]$. When the layers were polycrystalline through thickness ( $h$ $>1 \mu \mathrm{m}$ Fig. 2a-b), the textures were those expected of $\mathrm{Cu}$ and $\mathrm{Nb}$ when rolled alone. It would appear that in these coarser composites the bimetal interface has little influence.

When a single grain spanned the entire layer (below $600-1200 \mathrm{~nm}$ or so) (Fig. 2c) the textures sharpened significantly. The chosen orientations in ARB corresponded to a few select ideal rolling components [43]. The $\mathrm{Cu}$ crystals range from the $\mathrm{C}=$ $\{112\}<111>$ component to the $\mathrm{D}=\left\{\begin{array}{lll}4 & 4 & 11\end{array}\right\}<11118>$ component (differing by an $8^{\circ}$ tilt about the $\langle 110\rangle$ axis) and the $\mathrm{Nb}$ crystals are nearly locked on the $\mathrm{I}=$ $\{112\}<110>$ component, $\pm 5^{\circ}$. (Rolling components are expressed in the standard \{rolling plane $<<$ rolling direction $>$ nomenclature.) It was remarkable that these few orientations naturally emerged in extreme ARB straining when the number of possible ideal rolling orientations is substantially larger.

Such highly oriented textures in the $\mathrm{Cu}$ and $\mathrm{Nb}$ imply that a particular $\mathrm{Cu}-\mathrm{Nb}$ interface character is prevailing over the material. Using electron back scatter diffraction (EBSD) for phase and orientation mapping, it was shown that the $\mathrm{Cu}$ and $\mathrm{Nb}$ crystals preferred to be joined at their mutual $\{112\}$ planes and their $<111>\mathrm{Cu} \| \mid<110>\mathrm{Nb}$ directions were aligned, with a $\sim 10^{\circ}$ orientation spread (Fig. 3 ) [44].

Figure 3. Schematic of a predominant interface character that emerges in $\mathrm{Cu}-\mathrm{Nb}$ nanolayered composites after extremely large rolling strains.

Amazingly, this SPD multilayer became highly oriented much like a physical vapor deposited (PVD) $\mathrm{Cu}-\mathrm{Nb}$ multilayered [44-46]. In PVD foils, the $\mathrm{Cu}$ and $\mathrm{Nb}$ crystals develop a Kurdjumov-Sachs (KS) interface, with the $\{111\}_{\text {fcc }}$ plane joined to the $\{110\}_{\text {bcc }}$ plane at the interface and the $<110>$ fcc and $<111>$ bcc directions aligned inplane, i.e., $\{111\}<110>\mathrm{Cu} \|\{110\}<111>\mathrm{Nb}$ [47]. This interface has a relatively low formation energy $\left(576-586 \mathrm{~mJ} / \mathrm{m}^{2}\right.$ [48]. The prevailing ARB interface, $\{112\}<111>\mathrm{Cu}||\{112\}<110>\mathrm{Nb}$ interface, or $\mathrm{C}$-I in short, shares the same orientation relationship as the KS interface found in PVD $\mathrm{Cu}-\mathrm{Nb}$ foils but joins different interface planes and has a higher formation energy $\left(825 \mathrm{~mJ} / \mathrm{m}^{2}\right)$ [49] [50].

To determine if the C-I interface was stable with respect to straining, the analysis was repeated at layer thicknesses below $200 \mathrm{~nm}$ using more highly resolved microscopy techniques than standard EBSD $[51,52]$. The C-I interface and its close neighbor D-I interface (i.e., $\left\{\begin{array}{lll}4 & 11\end{array}\right\}<11118>\mathrm{Cu} \|\{112\}<110>\mathrm{Nb}$, which is tilted $8^{\circ}$ from C-I about the $\left\langle 110>_{\mathrm{fcc}}\right.$ ) prevailed in these finer layers (down to $30 \mathrm{~nm}$ ) as well. This result indicates that more straining created more C-I interface.

\subsection{When are preferred interfaces created?}


Bimetal interfaces are created in all stages of the ARB process (Fig. 2), and it is natural to wonder at which point do the predominant C-I and D-I interfaces begin to emerge. To address this question, bimetal interfaces within coarser, micron-layered ( $h=45 \mu \mathrm{m}, 20 \mu \mathrm{m}$, and $8 \mu \mathrm{m}$ ) ARB Cu-Nb composites were examined [53]. In these materials, several grains still spanned the layers. Yet as severe strains $\sim 4-6$ were required to make these samples, grain refinement within the $\mathrm{Cu}$ and $\mathrm{Nb}$ phases had already saturated to an average grain size of $D_{\text {sat }}=600 \mathrm{~nm}$. In accord, the length scale at which the composite layers become single-grain thick and highly oriented is approximately twice $D_{\text {sat, }}$ i.e., $\sim 600 \mathrm{~nm}-1200 \mathrm{~nm}$.

Within these composites, those grains joined to a dissimilar phase at a bimetal interface were identified as interface grains (Fig. 2b) and distinguished from the internal bulk grains, bounded only by grain boundaries. Using EBSD, it was found that the interface-grain-only texture was different than the bulk texture. More significantly, the former texture corresponded to the highly oriented texture of the nanolayered composites with layers below $600 \mathrm{~nm}$. This result indicates that SPD created preferred C-I to D-I interfaces even in the thicker layered composites.

\subsection{Preferred interface in crystals that slip and twin}

For some fcc metals, mediation of plastic deformation, whether by ordinary slip, partial dislocation slip, or deformation twinning is known to be strongly dependent on nanograin size $[29,54,55]$. In the ARB composites, such a crossover in deformation mechanisms was seen when examining the very finest of layers $h$. As the layers are refined below $50 \mathrm{~nm}$, the $\mathrm{Cu}$ phase begins to form deformation twins heterogeneously throughout the sample, reaching a volume fraction of $30 \%$ when $h$ $=10 \mathrm{~nm}$ [56]. For such fine Cu grain sizes, some deformation twinning is expected and has been observed in other nanostructured $\mathrm{Cu}$ systems and predicted numerically [29, 36, 55, 57, 58]. More interestingly, the interfaces intersected by the twins still stabilized in extreme straining. Specifically, the twin-oriented parts of the $\mathrm{Cu}$ crystals that were joined to the $\mathrm{Nb}$ crystals formed a distinctly different interface, narrowly distributed about the $\{110\}<001>\mathrm{Cu} \|\{112\}<110>\mathrm{Nb}$, or G-I interface [43, 59]. Thus, it is possible for naturally selected interfaces to emerge in metals that deform by slip and twinning.

\subsection{Understanding preferred SPD-created bimetal interfaces}

\subsection{Two variables common to stable interfaces}

To date, there is no standard theory to explain the development of a preferred interface character (C-I to D-I) in a nanocomposite produced via SPD. The key variables governing the stability of SPD-created interfaces have yet to be identified.

Evidently one variable pertains to crystallographic orientation stability during plastic deformation. The C-I interface and its equally stable near-neighbor, the D-I interface, join the C, D, and I orientations, which individually are known as ideal orientations in rolling. This means that they can plastically deform with negligible lattice reorientation. Building on this concept, we can define an interface that 
maintains its crystallographic character in rolling--an ideal interface character in rolling. More generally (for other deformation modes apart from rolling), the character of such an interface is plastically stable, where the crystals on both sides are deforming yet neither is reorienting, so the interface character is preserved. When either or both crystals reorient, then the interface character is not plastically stable. Using mesoscale modeling, the plastic stability of the C-I and D-I interfaces as well as others will be discussed shortly.

Considering plastic stability alone, however, cannot be sufficient for explaining the observed development a narrow distribution of interfaces about C-I in SPD. While not all possible pairs of $\mathrm{Cu}$ and $\mathrm{Nb}$ ideal rolling components would constitute a plastically stable interface $[39,43,60]$, the number of plastically stable interfaces is anticipated to be large since there are many ideal rolling components for $\mathrm{Cu}$ and for $\mathrm{Nb}$.

An additional factor must be intervening, which when combined with considerations of plastic stability leads to the observed down selection. It has been proposed that this other variable is interface formation energy [39]. Refinement via plastic deformation creates new interfacial area (Fig. 4). If the interface formation energy were sensitive to interface character, then the interface formation energy can play a role in the stability of an interface in mechanical deformation. For twophase material systems like $\mathrm{Cu}-\mathrm{Nb}, \mathrm{MD}$ simulations have shown that interface formation energy is very sensitive to interface character $[59,61]$. On this basis, it is hypothesized that SPD creates a preferred interface that is simultaneously plastically stable and has low formation energy.

Figure 4. Coupling of the plastic stability of an interface and the energy associated with forming the new interface area. The interface character is plastically stable when the crystals on both sides of the interface do not reorient with deformation.

At present, a model that couples changes in formation energy with changes in interface character as the two crystals on either side of the interface plastically deform does not exist. Consequently, to test this hypothesis, in the next section, we examine the plastic stability and formation energy for the same set of interfaces using two separate simulation techniques and place the two results on a map, which clearly identifies a regime of preferred interfaces.

\subsection{Calculating the plastic stability of an interface}

Crystal plasticity finite element (CPFE) simulations are employed to determine the plastic stability of an interface $[60,62]$. With this technique, the structures and crystallography of the grains, grain boundaries and bimetal interfaces can be directly represented (Fig. 5) [63]. CPFE is selected for this work because it captures spatially resolved displacement, stress and strain fields according to crystal plasticity at the subgrain scale [64-68]. The simulations account for the differences in elastic anisotropy, viscoplastic flow behavior, and crystallographic slip systems in 
$\mathrm{Cu}$ and $\mathrm{Nb}$. They also capture mesoscale heterogeneities in deformation, such as localization at the grain boundaries and interfaces, should they occur $[62,69]$.

Figure 5. Crystal plasticity finite element mesh models of a two-phase layered composite (a) model of a grain structure mapped from experimental data and (b) model of a bicrystal.

We study the same interface using CPFE in two different configurations. In one case, the layers on each side of the interface are polycrystalline, with more than one grain spanning the layer thickness. This material model contains both grain boundaries and bimetal interfaces (Fig. 5a). In the other case we model a $\mathrm{Cu}$-Nb bicrystal, comprised of two single crystals joined at a common interface (Fig. 5b). It is intended to represent the finer nanolayered composites exhibiting high-aspect-ratio grains (on average 33 for $\mathrm{Cu}$ and 88 for $\mathrm{Nb}[13,35]$ ) and layers that are spanned by one crystal. Further it best applies to behavior far from the grain boundaries. Both types of interface models are deformed under plane strain compression, an idealization of rolling. In accord with experimental observation, the present calculations assume that the bimetal interface and grain boundaries are codeforming.

To examine plastic stability we map the absolute lattice reorientation angle from the original starting orientation. Figure 6 compares the reorientation maps for four different interfaces (C-I, D-I, G-I and KS interfaces) within a bicrystal after large rolling reductions $(75 \%)$. In these maps, blue means small amounts of lattice reorientation $\left(<6^{\circ}\right)$, whereas yellow to orange correspond to large reorientations (> $25^{\circ}$ ). For the C-I and D-I interfaces created in SPD (Fig. 6a,b), the reorientations are minimal and homogeneous. We also find that the G-I interface (Fig. 6c), which develops in the parts of the material where the $\mathrm{Cu}$ crystals have twinned, is also stable. In contrast, for the KS interface $(\{111\}<110>\mathrm{Cu}||\{110\}<111>\mathrm{Nb})$, prevailing in the $\mathrm{Cu}-\mathrm{Nb}$ PVD nanolayered foils [44, 47], the reorientations are inhomogeneous and large (Fig. 6d). Thus, the PVD interface is not plastically stable in large-strain rolling. In fact, the bicrystal model predicts that the PVD interface reorients toward the C-I interface after large rolling reductions [60], which is a testament to the stability of the C-I interface. In agreement, texture measurements on rolled PVD Cu$\mathrm{Nb}$ nanolayers suggest a similar reorientation path [45].

Figure 6. Reorientation maps of bicrystals deformed in plane strain compression. The top crystal is $\mathrm{Cu}$ and the bottom crystal is $\mathrm{Nb}$. The starting interface orientation relationships for these bicrystals correspond to the (a) C-I interface and (b) D-I interface, which are predominant interfaces emerging from the ARB process, and the (c) G-I interface, a stable interface formed as a result of slip and twinning in the $\mathrm{Cu}$ phase, and the (d) KS interface, which is a low-energy interface characteristic of PVD Cu-Nb nanolayered composites. The crystallographic orientations are overlaid onto the original mesh seen in Fig. $5 \mathrm{~b}$. The deformed mesh is not shown. 
To investigate the additional effects of grain boundaries on the plastic stability of interface grains, we repeated the calculation for the same four $\mathrm{Cu}-\mathrm{Nb}$ interfaces but now as part of the interface formed between two polycrystalline layers (Fig. 5a). Figure 7 shows the corresponding reorientation maps using the same color scheme. We see that the KS interface is still plastically unstable (Fig. 7d). The C-I and the D-I interfaces (Figs. 7a,b), however, maintain good plastic stability in the polycrystalline environment, apart from some variation in misorientation near the grain boundaries. This result suggests that these interfaces, once created, can remain stable, as the layers are refined from spanning several grains to one grain through the thickness (Fig. 2e). Last, the G-I interface (Fig. 7c) maintains its stability as well, which sheds light on why deformation twins can continue to expand to micron dimensions with continued straining in the $\mathrm{Cu}$ layer, even though they lead to concomitant dramatic changes in the interface character from C-I to G-I.

Figure 7. Reorientation maps of two-phase grain structures deformed in plane strain compression. The top phase is $\mathrm{Cu}$ and the bottom phase is $\mathrm{Nb}$. The central embedded bicrystal outlined in yellow in Fig. 5a has a prescribed starting interface crystallography. In (a), the crystallography corresponds to the C-I interface and in (b) the D-I interface. Interfaces in (a) and (b) are predominant interfaces emerging from the ARB process. In (c) the crystallography corresponds to the G-I interface, a stable interface formed as a result of slip and twinning in the $\mathrm{Cu}$ phase, and in (d) the KS interface, which is a low-energy interface characteristic of PVD Cu-Nb nanolayered composites. The crystallographic orientations are overlaid onto the original mesh seen in Fig. 5a. The deformed mesh is not shown. The legend is identical to that shown in Fig. 6.

To quickly assess the plastic stability of several interfaces, we define a plastic stability parameter $\omega$.

$$
\omega=\exp \left(-\frac{\Delta \Omega_{f c c}}{\Delta \varepsilon}\right) \exp \left(-\frac{\Delta \Omega_{b c c}}{\Delta \varepsilon}\right)
$$

where $\Delta \Omega_{f c c}$ and $\Delta \Omega_{b c c}$ are the amount of reorientation (as an angle in radians) from the initial orientation of the crystals on each side of the interface after $\Delta \varepsilon$ strain. (A similar form for Eq. (1) can be used for the rate of reorientation [62].) A value of 1 is considered perfectly stable, which means that both $\Delta \Omega_{f c c}$ and $\Delta \Omega_{b c c}$ are zero. A value $<1$ means that the interface character has changed as a result of a finite reorientation in either crystal. As a practical bound, we allow for small lattice reorientations $\left(<10^{\circ}\right)$ over large strains by arbitrarily considering interfaces with values $\omega>0.8$ as plastically stable interfaces.

\subsection{Coupling plastic stability and the free energy of an interface}

In addition to the plastic stability parameter, $\omega$, the interface formation energy, $\gamma$, is also considered. Previously, MD simulation, using reliable potentials for $\mathrm{Cu}, \mathrm{Nb}$, and their cross-interactions, estimated $\gamma$ for the same interfaces treated in CPFE [39, 59, 61]. It was shown that $\gamma$ of an interface is sensitive to its character. Accordingly, the influence of $\gamma$ on crystal deformation could be strong. 
To determine how the interfaces compare with respect to these two variables, the $\omega$ and $\gamma$ for several test interfaces were calculated and plotted on a map of $\omega$ vs. $\gamma$ in Fig. 8 [39]. According to our hypothesis, interfaces expected to emerge from severe deformation would have a low $\gamma$ and high $\omega$ and thus would lie in the upper lefthand corner of this map, labeled the 'stable region'. The test interfaces are marked by red symbols. The interfaces that were created by SPD and remained stable in straining are marked using black triangles. We note that they fall exclusively in the stable region of the map.

Figure 8. Map of crystallographic stability of interfaces in plastic deformation: plastic stability vs. interface formation energy $[39,60]$. Interface formation energy is normalized by the interface formation energy of a $\mathrm{Cu}-\mathrm{Nb}$ Kurdjumov-Sachs interface. Squares represent interfaces created by pairing orientations from the stable orientation distribution (i.e., well developed rolling texture for the monolithic, single-phase metal) of the deformed crystals. Circles represent interfaces formed when one or both orientations do not belong to the theoretical single-phase rolling texture. Interfaces with black triangles represent interface characters observed before the onset of twinning and those with black stars represent interface characters observed after the onset of twinning. Taken with permissions from [43].

The test interfaces that lie outside the stable region in the lower left-hand region include those found in $\mathrm{Cu}-\mathrm{Nb}$ PVD foils: the KS and its close relative, the NishiyamaWasserman interface [21]. Although they have much lower formation energy, $\gamma$, than those observed, they fall outside the stable region because they are plastically unstable (low $\omega$ ).

Other test interfaces lying in the unstable region found in the upper right-hand corner corresponded to those that combine $\mathrm{Cu}$ and $\mathrm{Nb}$ ideal orientations in rolling. These pairs were in fact even more plastically stable than the emergent interfaces, C-I and D-I. However, the map implies that they were either not created or unstable in rolling strains because they form high-energy interfaces (high $\gamma$ ).

The map provides valuable insight into which interfaces are stable in extreme strains, and it is sufficiently general to apply to other deformation modes and twophase material systems. Most importantly, the map opens up the possibility that SPD techniques can be used to synthesize stable interfaces.

\subsection{Areas for mesoscale modeling}

Investigation of interface effects on microstructural evolution is a wide-open research topic for both mesoscale experimental and materials modeling exploration. Before concluding, we discuss a few areas in mesoscale modeling that have great potential to contribute to our basic scientific understanding.

A prime example is given by the above study, which revealed a possible regime of crystallographically stable interfaces that have two aspects in common: being plastically stable in severe plastic deformation and having low formation energy 
(Fig. 8). To fully understand and predict the effect of interfaces on microstructural evolution, a mesoscale model is needed that couples the penalties or advantages associated with changes in interface formation energy, $\gamma$, with plastically driven changes in interface character.

\subsection{Mesoscale modeling for microstructural evolution}

Explaining how grain refinement occurs is one unsolved problem in SPD that is ideally suited for mesoscale modeling. In the foregoing study of ARB two-phase materials, we observe that $D_{\text {sat }}$ is reached while the layers are still polycrystalline (Fig. 2b-c). When the bimetal interface spacing, $h$, approaches $D_{\text {sat }}$ (Fig. $2 \mathrm{~d}$ ) the layers are one-grain thick. How do the layers transition from polycrystalline to single crystalline? Do the interface grains remain at the interface while the bulk grains disappear? Are the bulk grains constantly replacing the interface grains or are the interface grains being re-generated? These are questions that call for mesoscale characterization and modeling.

As a first attempt toward understanding this phenomenon, we return to the polycrystalline layered CPFE model in Figs. 5a and 7 and examine the influence of grain neighborhood on the plastic stability of the interface grains. Figure 9 shows reorientation maps after $100 \%$ rolling strain of a C-I bicrystal in two different but likely grain neighborhoods: (a) highly oriented neighborhood, similar to the interface orientations, or a (b) sampled realization of rolling texture. In both cases, the interface grains exhibit similar responses. The interface grains appear to be plastically stable regardless of the stability of the neighborhood. We also find large reorientations near the grain boundaries but lower ones at the bimetal interfaces. These results suggest that once the stable interface orientations are established, they do not leave this state. Based on these results, we speculate that $h$ refines, the bulk grains in between are reorienting to coincide with the more stable orientations at the interface. This would result in an apparent disappearance of the bulk grain orientations and persistence of the interface grain orientations as layer thicknesses become finer with rolling deformation.

Figure 9. Reorientation maps of two-phase grain structure deformed in plane strain compression. The top phase is $\mathrm{Cu}$ and the bottom phase is $\mathrm{Nb}$. The central embedded bicrystal outlined in yellow in Fig. 5a has a prescribed starting interface crystallography corresponding to the C-I interface in (a) a highly oriented neighborhood of $\mathrm{Cu}$ grains and $\mathrm{Nb}$ grains oriented close to the $\mathrm{C}$ and $\mathrm{I}$ texture components, respectively and in (b) a neighborhood sampled from a typical rolling texture for $\mathrm{Cu}$ and $\mathrm{Nb}$. The crystallographic orientations are overlaid onto the original mesh seen in Fig. 5a. The deformed mesh is not shown.

Although the above example has provided some insight, there is much more modeling work to do. Mesoscale models not only need to be developed to predict when and where plasticity creates grain boundaries and interfaces but also to predict when and where plasticity stops creating grain boundaries and interfaces (recovery, migration). The problem of nanostructuring in two-phase metals in SPD is a perfect example of a problem situated in this gap. 


\subsection{Mesoscale modeling for nanoscale plasticity}

Nanoscale plasticity conveys the possibility that plastic deformation of nanocrystals is different from ordinary slip. Indeed, the fine nanoscale dimensions and prevailing grain boundary-dislocation interactions are anticipated to significantly modify slip behavior from that observed in coarser-sized crystals. Some examples of deformation mechanisms believed to dominant in nanocrystals include threading of dislocations through a layer (confined layer slip [16, 70], transmission of a dislocation from one crystal to another across a boundary (slip transmission [27, 30, 71, 72], interfacial sliding [21], and unhindered glide of a dislocation emitted from one interface and absorbed at another [73-75]. These mechanisms are not expected to be active in traditional metals and conventional crystal plasticity models do not account for them.

As a means of describing how mesoscale modeling could help in this regard, we focus on one of the nano-enabled mechanisms mentioned above: slip transmission. It is well known that slip transmission can lead to important changes in nanomaterial strength and ductility. In relation to macroscopic data, predominance of slip transmission has been tied to the attainment of peak or saturation strengths in hardness with decreasing layer thickness $[11,12,16,76]$. In atomistic simulation, the ease of transmission has been correlated with shock resistance [26], and it has been related to the onset of plastic instabilities in nanopillar compression [20].

It can easily be envisioned that in interface-dominant, nanostructured metals, slip transmission can have a profound impact on microstructural and texture evolution during deformation. The interiors of nanoscale grains in nanostructured metals typically are nearly free of defects and thus lack sufficient sources for mobile dislocations. Consequently, transmission of dislocations from one nanocrystal to another across an interface can be a chief source of dislocations needed to carry the plasticity. However, both experimental and numerical modeling studies find that in most cases, slip transmission across a boundary is difficult. Thus, dislocations are not expected to freely pass from one crystal to another across an interface via any pathway. In fact, many studies have been devoted to defining the special relationships between the preferred incoming and outgoing slip systems through which dislocations prefer to use and assessing the large driving stresses required for these maneuvers [20,31, 64, 71, 72, 77]. Consequently, when slip transmission across interfaces is the main source for mobile dislocations, sustaining plasticity in the adjoining crystals can become constrained. Moreover, slip activity in nanocrystals can be significantly altered by the interface and hence microstructure and texture evolution noticeably affected.

Modeling efforts and most experimental studies have not yet considered how slip transmission affects texture evolution. Recently, a first attempt was made to examine the effect of slip transmission on slip activity in the adjoining crystals and in turn the evolution of interface character. To this end, a model for slip transmission across a bimetal interface was developed and incorporated into the same two-phase CPFE model described earlier [78]. A simple criterion for slip 
transmission through a given pair of slip systems across the interface was employed and based solely on the degree of alignment of their slip planes and slip directions and whether or not the local mechanical driving forces supported the flux into and out of the interface [31].

Figure 10 shows reorientation maps for the C-I and G-I interface with sliptransmission-dominated slip as calculated from this model. To see the effects of slip transmission, these can be directly compared to their maps in Fig. $6 \mathrm{a}$ and $6 \mathrm{~d}$ calculated with ordinary slip. In all cases, we found that slip transmission across the interface significantly altered the slip activity. The alteration causes the C-I interface (and D-I interface as well) to become unstable (reorientations greater than $12^{\circ}$ ). In contrast, G-I interface maintained its character in rolling. The results show that the G-I interface is stable with and without slip transmission-dominated slip, even though the slip activity in these two cases is not the same.

Figure 10. Reorientation maps of bicrystals deformed in plane strain compression and with slip activity controlled by slip transmission. The top crystal is $\mathrm{Cu}$ and the bottom crystal is $\mathrm{Nb}$. The starting interface orientation relationships for these bicrystals correspond to the (a) C-I interface and (b) D-I interface, which are predominant interfaces emerging from the ARB process, and the (c) G-I interface. The crystallographic orientations are overlaid onto the original mesh seen in Fig. 5a. The deformed mesh is not shown. The legend is the same one as that shown in Fig. 6.

The slip-transmission-dominated calculations assume the highly constraining situation in which only slip systems belonging to favorable slip transmission pathways are active [78]. In actuality, the constraint may be less severe as the interface can supply dislocations by other means (e.g., direct nucleation at triple junctions). Coupling all relevant interface driven plasticity phenomena is a future task especially well-suited for mesoscale modeling approaches.

\subsection{Conclusions}

Recently it was shown that thermally stable, ultra-strong "bulk" nanolayered composites can be made with morphological and microstructural control via severe plastic deformation SPD $[13,35,39]$. These SPD techniques are non-equilibrium thermomechanical processes and involve imposing tremendously large plastic strains to refine the grain size and layer spacing by several orders of magnitude while creating a preferred, predominant bimetal interface.

Future integration of such superior nanostructured metal composites encounters the common challenges of quantifying and predicting microstructure-property relationships. Two fundamental aspects that impact these relationships are 1) the effect of interfaces on the evolution of grain structure that accompanies plasticity and 2) the effect of plasticity on the evolution of the interface. These two processes are intimately coupled and understanding this interrelationship requires characterization and modeling at the mesoscale. 
As a focal point, we discussed the development of bimetal interfaces in severe plastic deformation by accumulative roll bonding (ARB) using mesoscale techniques. We discussed how a few preferred interface characters are naturally selected by the extreme plastic deformation and identified two common variables that appear to govern the down-selection process. A map is presented that exposes a regime of stable interfaces. This radical concept indicates that it is possible to use ARB, as well as other SPD processes, to manufacture bulk nanocomposites with specific classes of interfaces. These plasticity-driven interfaces can potentially be designed for exceptional performance in a wide range of structural, functional, and biological applications.

\section{Acknowledgements}

The authors gratefully acknowledge support by the Center for Materials at Irradiation and Mechanical Extremes, an Energy Frontier Research Center funded by the US Department of Energy, Office of Science, Office of Basic Energy Sciences under Award Number 2008LANL1026. Los Alamos National Laboratory is operated by Los Alamos National Security LLC under DOE Contract DE AC52 06NA25396.

\section{References}

[1] K. Lu, Science, 328 (2010) 319-320.

[2] C.A. Handwerker, T.M. Pollock, Jom, 66 (2014) 1321-1341.

[3] W.Z. Han, E.K. Cerreta, N.A. Mara, I.J. Beyerlein, J.S. Carpenter, S.J. Zheng, C.P. Trujillo, P.O. Dickerson, A. Misra, Acta Materialia, 63 (2014) 150.

[4] W. Han, M.J. Demkowicz, N.A. Mara, E. Fu, S. Sinha, A.D. Rollett, Y. Wang, J.S. Carpenter, I.J. Beyerlein, A. Misra, Advanced Materials, 25 (2013) 6975-6979.

[5] H. Gleiter, Acta Materialia, 56 (2008) 5875-5893.

[6] T. Nizolek, N.A. Mara, J. Avallone, I.J. Beyerlein, T.M. Pollock, Advanced Engineering Materials, In Press (2014).

[7] N.A. Mara, I.J. Beyerlein, Journal of Materials Science, 49 (2014) 6497-6516.

[8] I.J. Beyerlein, A. Caro, M.J. Demkowicz, N.A. Mara, A. Misra, B.P. Uberuaga, Materials Today, 16 (2013) 443.

[9] I.J. Beyerlein, N.A. Mara, J.S. Carpenter, T. Nizolek, W.M. Mook, T.A. Wynn, R.J. McCabe, J.R. Mayeur, K. Kang, S. Zheng, J. Wang, T.M. Pollock, Journal of Materials Research, 28 (2013) 1799-1812.

[10] N.A. Mara, D. Bhattacharyya, P. Dickerson, R.G. Hoagland, A. Misra, Applied Physics Letters, 92 (2008) 231901-231903.

[11] M.A. Monclus, S.J. Zheng, J.R. Mayeur, I.J. Beyerlein, N.A. Mara, T. Polcar, J. Llorca, J.M. Molina-Aldareguia, Applied Physics Letters Materials, 1 (2014) 052103.

[12] A. Misra, Mechanical Behavior of Metallic Nanolaminates, in: A.J. Hill , R.H.J. Hannink (Eds.) Nanostructure Control of Materials, Woodhead Publishing Co., UK, 2006, pp. 146-176.

[13] S. Zheng, J.S. Carpenter, R.J. McCabe, I.J. Beyerlein, N.A. Mara, Scientific Reports, 4 (2014) 4226. 
[14] J.S. Carpenter, S.J. Zheng, R.F. Zhang, S.C. Vogel, I.J. Beyerlein, N.A. Mara, Philosophical Magazine, 93 (2013) 718-735.

[15] F. Spaepen, D.Y.W. Yu, H. Huang, Nato Advanced Science Institutes Series, Series E, Applied Sciences, 367 (2000) 127-141.

[16] A. Misra, J.P. Hirth, R.G. Hoagland, Acta Materialia, 53 (2005) 4817-4824.

[17] E. Martinez, A. Caro, I.J. Beyerlein, Physical Review B, 90 (2014) 054103.

[18] R.J. McCabe, I.J. Beyerlein, J.S. Carpenter, N.A. Mara, Nature Communications, 5 (2014) 3806.

[19] S.J. Zheng, I.J. Beyerlein, J. Wang, J.S. Carpenter, W.Z. Han, N.A. Mara, Acta Materialia, 60 (2012) 5858-5866.

[20] S.J. Zheng, J. Wang, J.S. Carpenter, W.M. Mook, P.O. Dickerson, N.A. Mara, I.J. Beyerlein, Acta Materialia, 79 (2014) 282-291.

[21] J. Wang, C.Z. Zhou, I.J. Beyerlein, S. Shao, Jom, 66 (2014) 102-113.

[22] A. Hunter, I.J. Beyerlein, APL Materials, 1 (2013) 032109.

[23] N. Abdolrahim, H.M. Zbib, D.F. Bahr, International Journal of Plasticity, 52

(2014) 33-50.

[24] I.J. Beyerlein, J. Wang, R. Zhang, APL Materials, 1 (2013) 032112.

[25] I.J. Beyerlein, J. Wang, R. Zhang, Acta Materialia, 61 (2013) 7488-7499.

[26] R.F. Zhang, T.C. Germann, J. Wang, X.Y. Liu, I.J. Beyerlein, Scripta Materialia, 68

(2012).

[27] J. Wang, R.G. Hoagland, J.P. Hirth, A. Misra, Acta Materialia, 56 (2008) 31093119.

[28] R.F. Zhang, T.C. Germann, X.Y. Liu, J. Wang, I.J. Beyerlein, Acta Materialia, 79 (2014) 74-83.

[29] I.J. Beyerlein, X. Zhang, A. Misra, Annual Review of Materials Research, 44 (2014) 329-363.

[30] J. Wang, I.J. Beyerlein, N.A. Mara, D. Bhattacharyya, Scripta Materialia, 64 (2011) 1083-1086.

[31] I.J. Beyerlein, J. Wang, K. Kang, S.J. Zheng, N.A. Mara, Materials Research Letters, 1 (2013) 89-95.

[32] I.J. Beyerlein, L.S. Toth, Progress in Materials Science, 54 (2009) 427-510.

[33] U.F. Kocks, C.N. Tome, H.-R. Wenk, Texture and Anisotropy: Preferred orientations in polycrystals and their effect on material properties, Cambridge University Press, Cambridge, 1998.

[34] R.Z. Valiev, T.G. Langdon, Progress in Materials Science, 51 (2006) 881-981.

[35] J.S. Carpenter, R.J. McCabe, S.J. Zheng, T.A. Wynn, N.A. Mara, I.J. Beyerlein, Metallurgical and Materials Transactions a-Physical Metallurgy and Materials Science, 45A (2014) 2192-2208.

[36] I.J. Beyerlein, N.A. Mara, D. Bhattacharyya, D.J. Alexander, C.T. Necker, International Journal of Plasticity, 27 (2011) 121-146.

[37] K. Yasuna, M. Terauchi, A. Otsuki, K.N. Ishihara, P.H. Shingu, Materials Science and Engineering A, 285 (2000) 412-417.

[38] J.S. Carpenter, T. Nizolek, N.A. Mara, I.J. Beyerlein, Materials Research Letters, In press (2014). 
[39] I.J. Beyerlein, J.R. Mayeur, S.J. Zheng, N.A. Mara, J. Wang, A. Misra, Proceedings of the National Academy of Sciences of the United States of America, 111 (2014) 43864390.

[40] I.J. Beyerlein, N.A. Mara, J. Wang, J.S. Carpenter, S.J. Zheng, W.Z. Han, R.F. Zhang, K. Kang, T. Nizolek, T.M. Pollock, Jom, 64 (2012) 1192-1207.

[41] O. Engler, V. Randle, Introduction to texture analysis, CRC Press, Taylor and Francis Group, Boca Raton, FL, 2010.

[42] J.S. Carpenter, S.C. Vogel, J.E. Ledonne, D.L. Hammon, I.J. Beyerlein, N.A. Mara, Acta Materialia, 60 (2012) 1576-1586.

[43] I.J. Beyerlein, J.R. Mayeur, R.J. McCabe, S.J. Zheng, J.S. Carpenter, N.A. Mara, Acta Materialia, 72 (2014) 137-147.

[44] S.B. Lee, J.E. LeDonne, S.C.V. Lim, I.J. Beyerlein, A.D. Rollett, Acta Materialia, 60 (2012) 1747-1761.

[45] K. Al-Fadhalah, C.N. Tome, A.J. Beaudoin, I.M. Robertson, J.P. Hirth, A. Misra, Philosophical Magazine, 85 (2005) 1419-1440.

[46] A. Misra, J.P. Hirth, R.G. Hoagland, J.D. Embury, H. Kung, Acta Materialia, 52 (2004) 2387-2394.

[47] K. Yu-Zhang, J.D. Embury, K. Han, A. Misra, Philosophical Magazine, 88 (2008) 2559-2567.

[48] M.J. Demkowicz, R.G. Hoagland, J.P. Hirth, Physical Review Letters, 100 (2008) 136102-136101-136104.

[49] M.J. Demkowicz, L. Thilly, Acta Materialia, 59 (2011) 7744-7756.

[50] K. Kang, J. Wang, I.J. Beyerlein, Journal of Applied Physics, 111 (2012) 053531.

[51] J.S. Carpenter, R.J. McCabe, I.J. Beyerlein, T.A. Wynn, N.A. Mara, Journal of Applied Physics, 113 (2013).

[52] J.S. Carpenter, X. Liu, A. Darbal, N.T. Nuhfer, R.J. McCabe, S.C. Vogel, J.E. Ledonne, A.D. Rollett, K. Barmak, I.J. Beyerlein, N.A. Mara, Scripta Materialia, 67 (2012) 336339.

[53] J.S. Carpenter, R.J. McCabe, J.R. Mayeur, N.A. Mara, I.J. Beyerlein, Advanced Engineering Materials, (2014).

[54] J.W. Christian, S. Mahajan, Progress in Materials Science, 39 (1995) 1-57.

[55] Y.T. Zhu, X.Z. Liao, X.L. Wu, Prog. Mater. Sci., 57 (2012) 1-62.

[56] R.J. McCabe, I.J. Beyerlein, J.S. Carpenter, N.A. Mara, Nature Communications, 5 (2014) 3806.

[57] W.Z. Han, J.S. Carpenter, J. Wang, I.J. Beyerlein, N.A. Mara, Applied Physics Letters, 100 (2012) 011911.

[58] Y.H. Yue, P. Liu, Q.S. Deng, E. Ma, Z. Zhang, X.D. Han, Nano Letters, 12 (2012) 4045-4049.

[59] S. Zheng, I.J. Beyerlein, J.S. Carpenter, K. Kang, J. Wang, W. Han, N.A. Mara, Nature Communications, 4 (2013).

[60] J.R. Mayeur, I.J. Beyerlein, C.A. Bronkhorst, H.M. Mourad, B.L. Hansen, International Journal of Plasticity, 48 (2013) 72.

[61] K. Kang, J. Wang, S.J. Zheng, I.J. Beyerlein, Journal of Applied Physics, 112 (2012) 073501 (073510 pp.).

[62] J. Mayeur, I. Beyerlein, C. Bronkhorst, H. Mourad, Materials, 7 (2014) 302-322. 
[63] B.L. Hansen, J.S. Carpenter, S.D. Sintay, C.A. Bronkhorst, R.J. McCabe, J.R. Mayeur, H.M. Mourad, I.J. Beyerlein, N.A. Mara, S.R. Chen, G.T. Gray, International Journal of Plasticity, 49 (2013) 71-84.

[64] F. Roters, P. Eisenlohr, L. Hantcherli, D.D. Tjahjanto, T.R. Bieler, D. Raabe, Acta Materialia, 58 (2010) 1152-1211.

[65] M. Knezevic, B. Drach, M. Ardeljan, I.J. Beyerlein, Comput Method Appl M, 277 (2014) 239-259.

[66] T.R. Bieler, P. Eisenlohr, F. Roters, D. Kumar, D.E. Mason, M.A. Crimp, D. Raabe, International Journal of Plasticity, 25 (2009) 1655-1683.

[67] L. Wang, R.I. Barabash, Y. Yang, T.R. Bieler, M.A. Crimp, P. Eisenlohr, W. Liu, G.E. Ice, Metallurgical and Materials Transactions a-Physical Metallurgy and Materials Science, 42A (2011) 626-635.

[68] M. Anahid, M.K. Samal, S. Ghosh, Journal of the Mechanics and Physics of Solids, 59 (2011) 2157-2176.

[69] N. Jia, F. Roters, P. Eisenlohr, D. Raabe, X. Zhao, Acta Materialia, 61 (2013) 4591 4606.

[70] J.D. Embury, J.P. Hirth, Acta Metallurgica Et Materialia, 42 (1994) 2051-2056.

[71] E. Werner, W. Prantl, Acta Metallurgica Et Materialia, 38 (1990) 533-537.

[72] L. Patriarca, W. Abuzaid, H. Sehitoglu, H.J. Maier, Mat Sci Eng a-Struct, 588

(2013) 308-317.

[73] K.S. Kumar, S. Suresh, M.F. Chisholm, J.A. Horton, P. Wang, Acta Materialia, 51 (2003) 387-405.

[74] K.S. Kumar, H. Van Swygenhoven, S. Suresh, Acta Materialia, 51 (2003) 57435774.

[75] X.L. Wu, Y.T. Zhu, Y.G. Wei, Q. Wei, Physical Review Letters, 103 (2009).

[76] S.L. Lehoczky, Journal of Applied Physics, 49 (1978) 5479-5485.

[77] T.C. Lee, I.M. Robertson, H.K. Birnbaum, Scripta Metallurgica, 23 (1989) 799803.

[78] J.R. Mayeur, I.J. Beyerlein, C.A. Bronkhorst, H.M. Mourad, International Journal of Plasticity, (2014). 

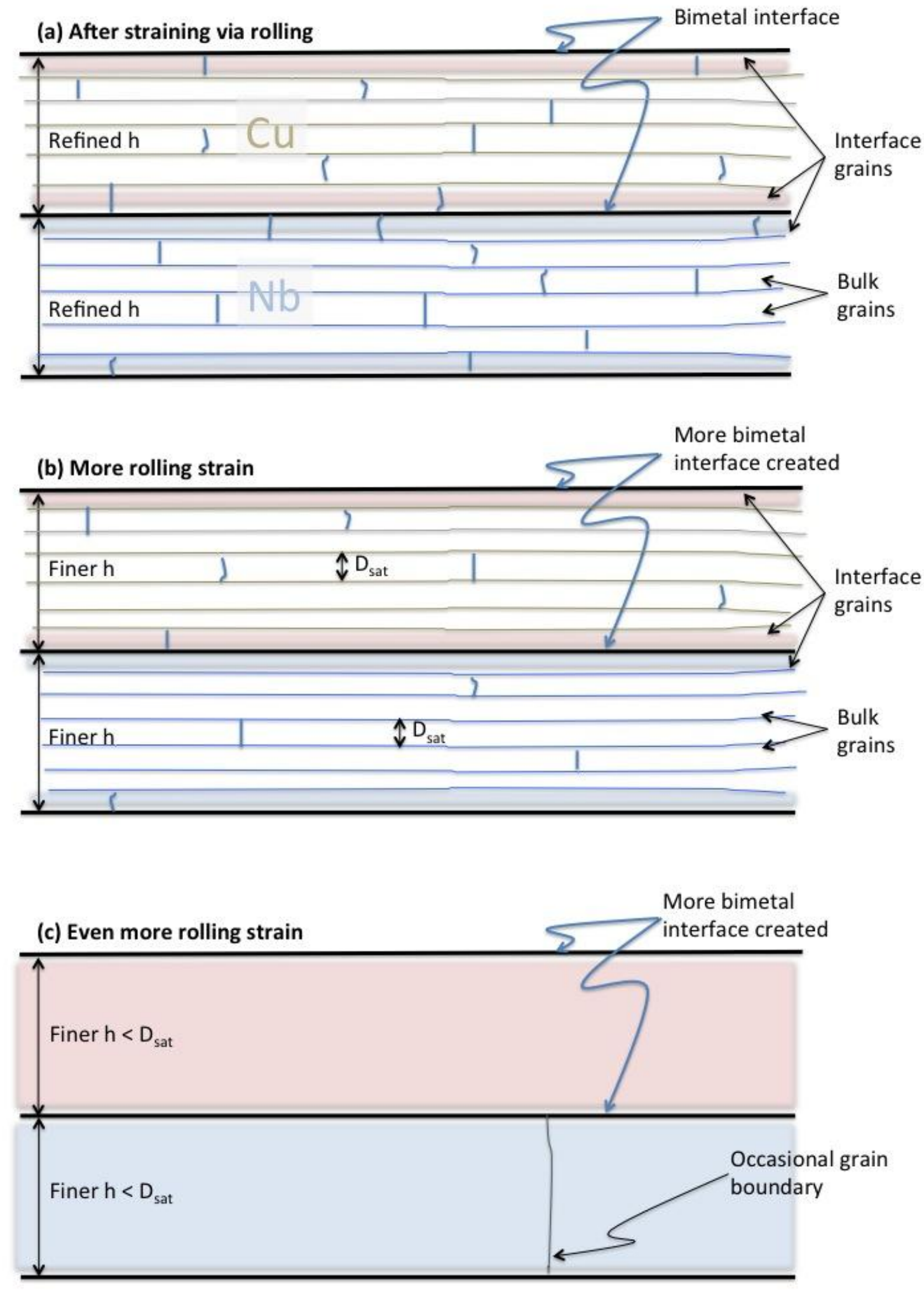
Figure3

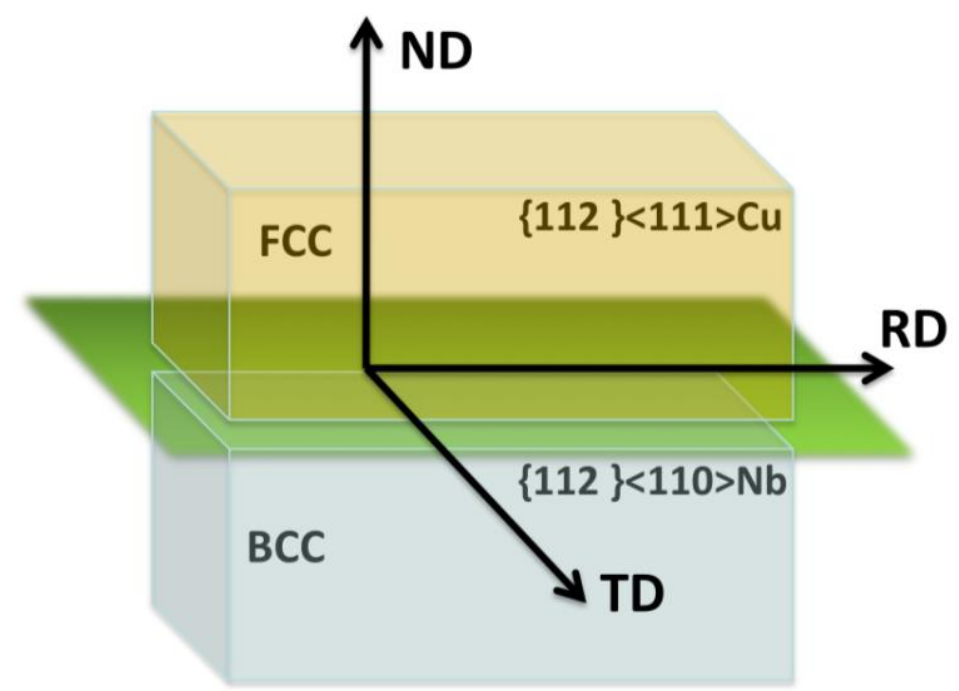


Figure4
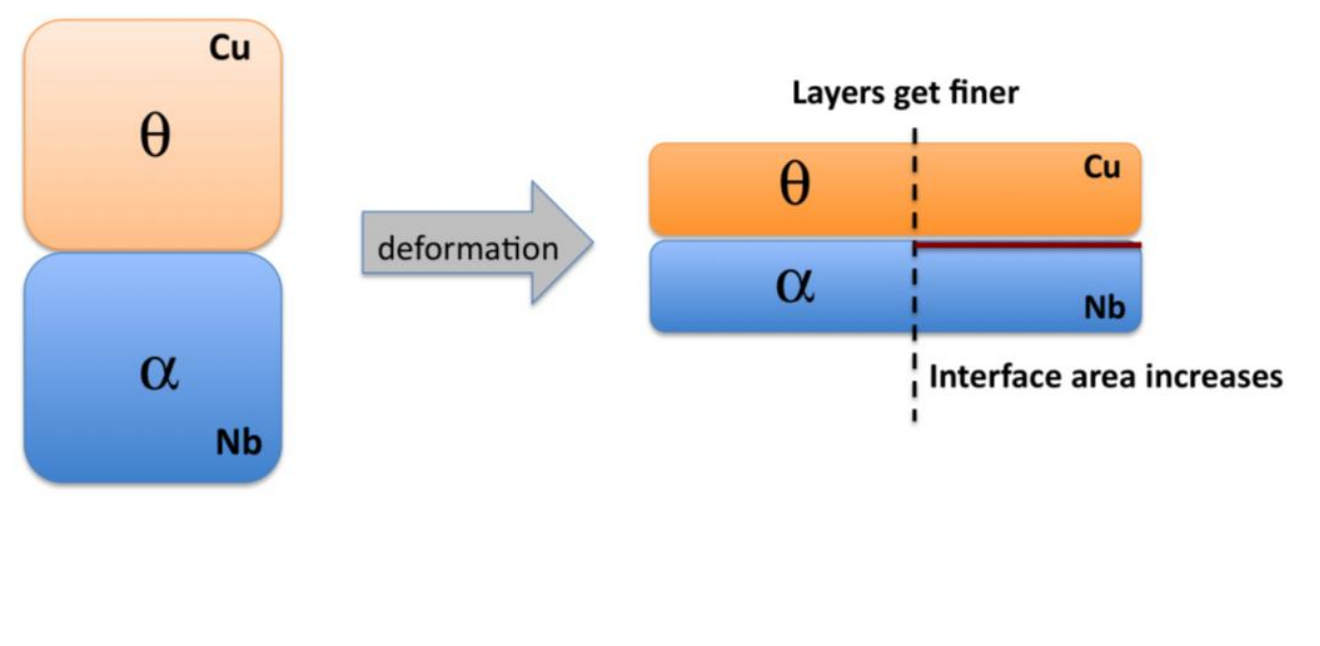

Interface area increases

Layers get finer

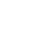
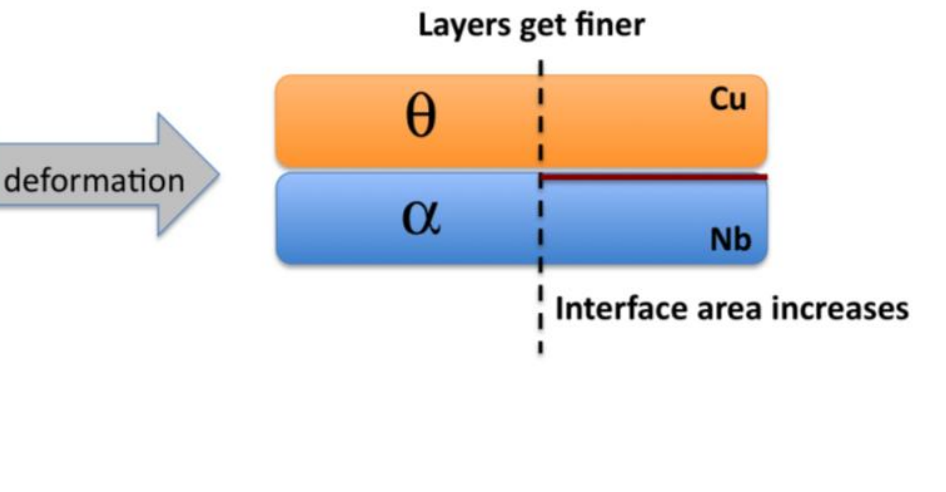

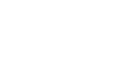

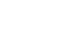

$\sqrt{2}+x^{2}$ (n)

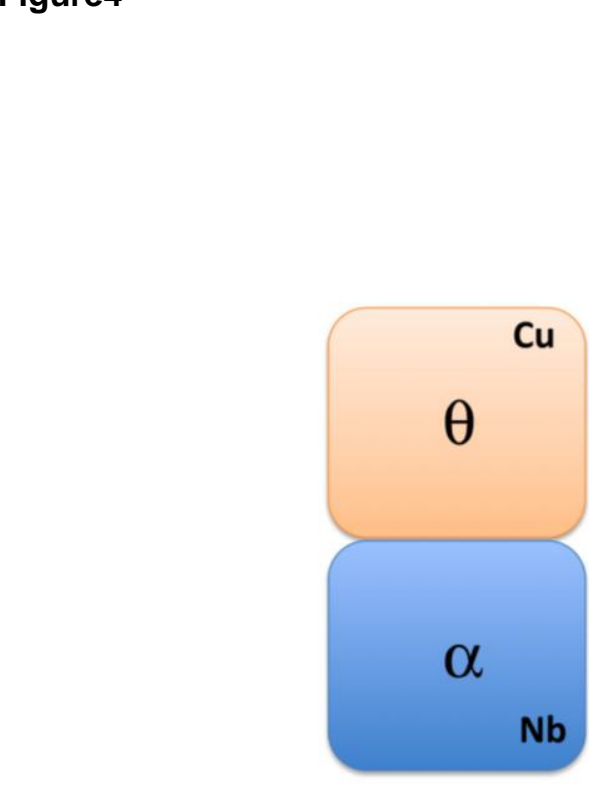

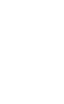
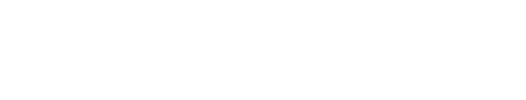

$$
\text { . }
$$

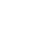

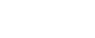

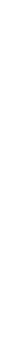



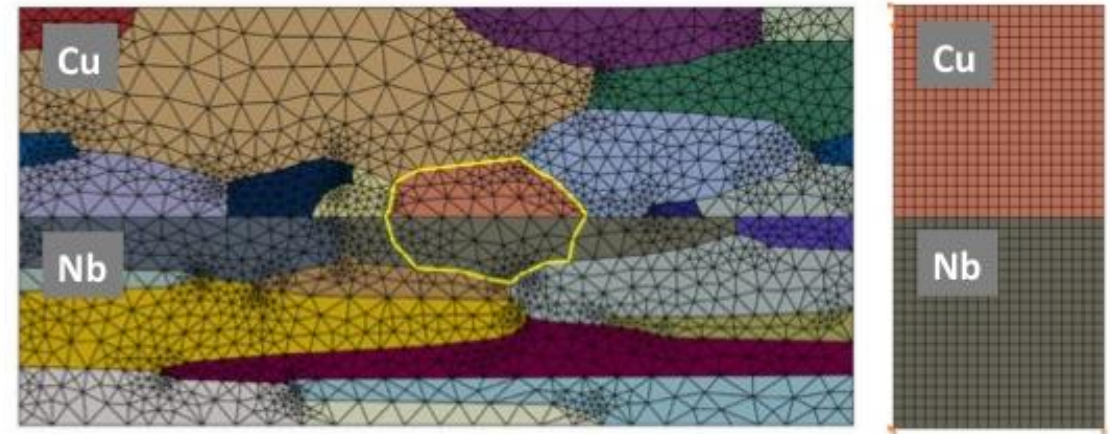

(a)

(b)

Figures
. 


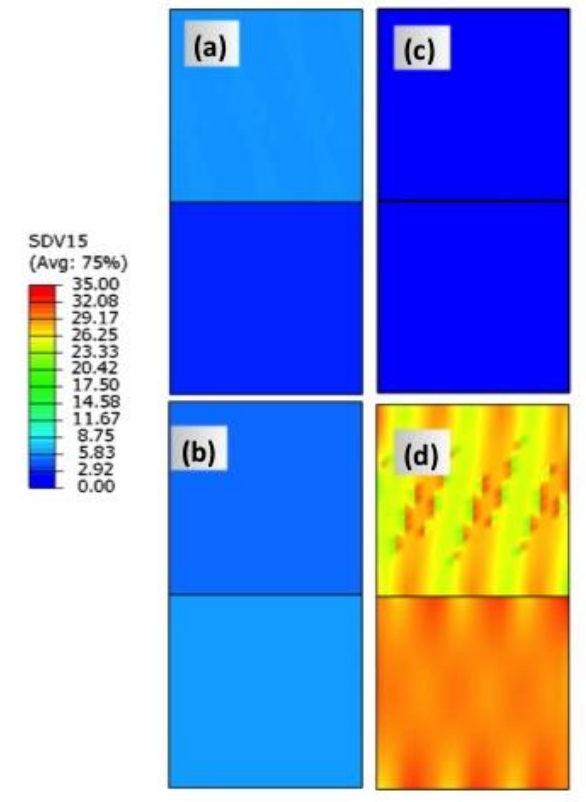



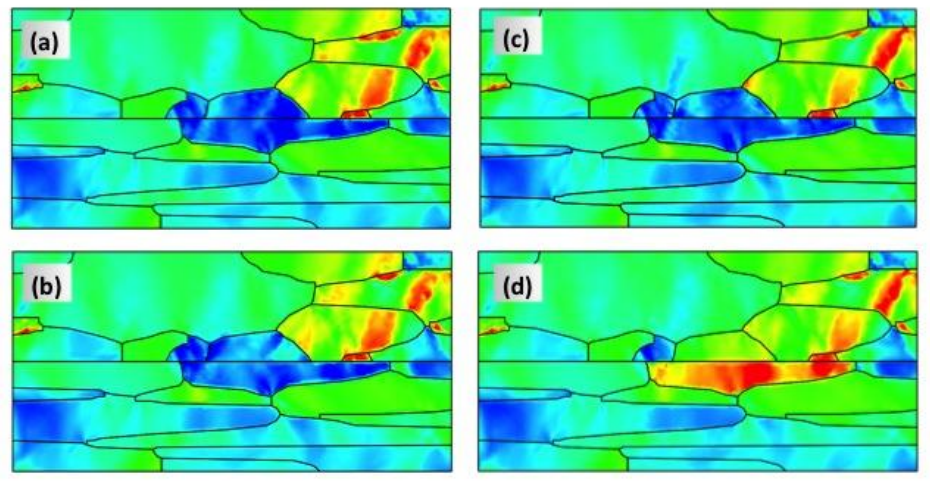


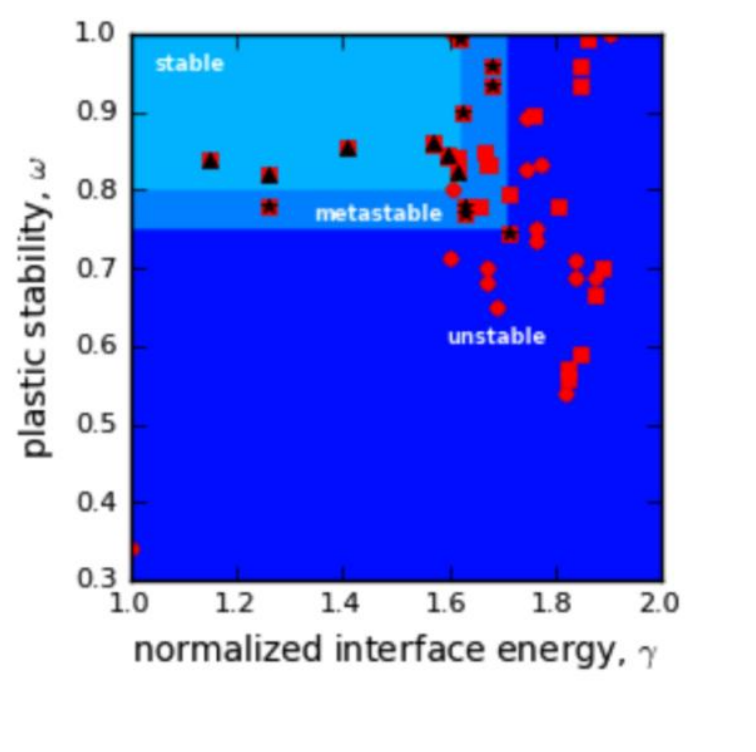

Figure8

rmalized interface energy, 7

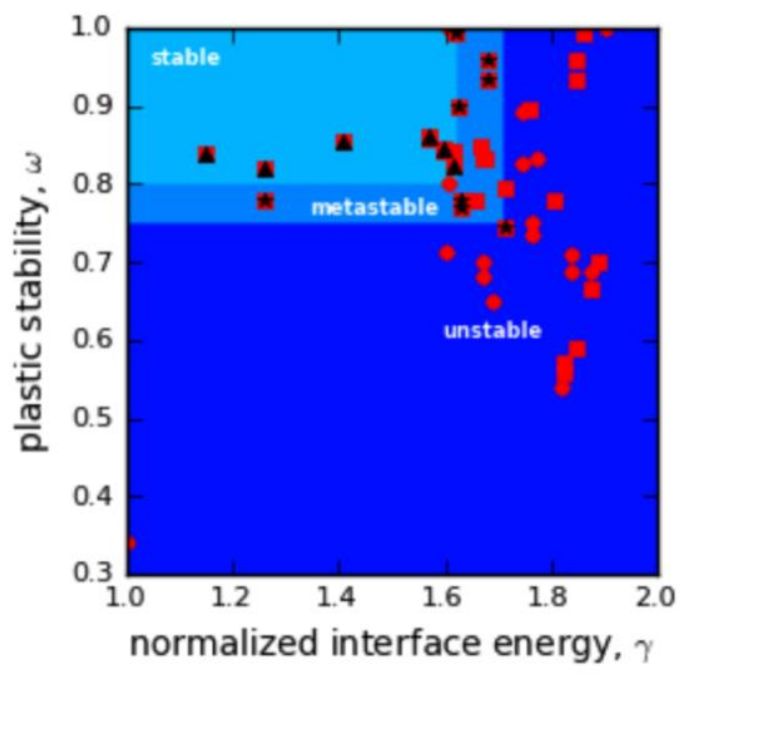




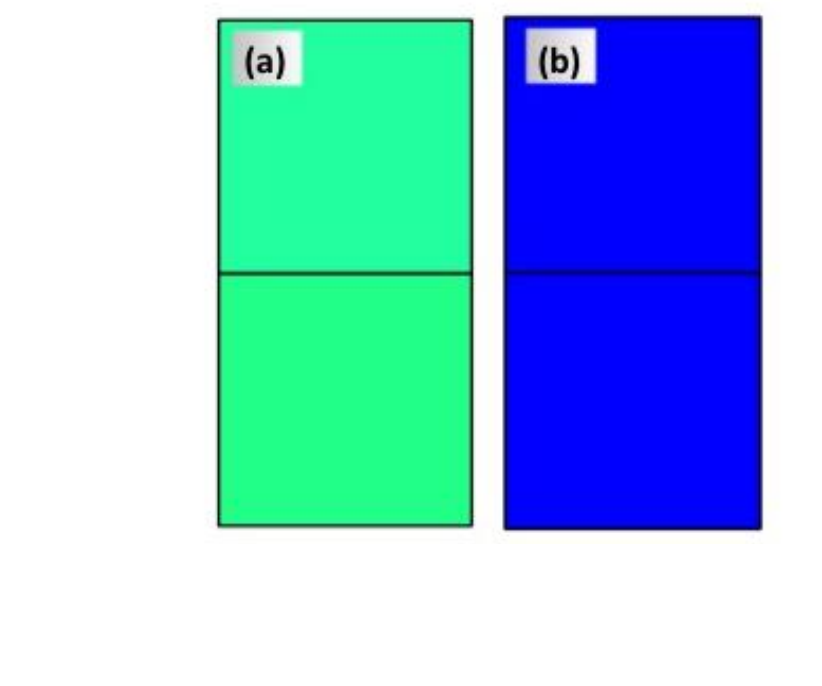

\begin{tabular}{l} 
Figure10 \\
$\qquad$\begin{tabular}{|l|l|}
\hline (a) \\
\hline \\
\end{tabular} \\
\hline
\end{tabular}
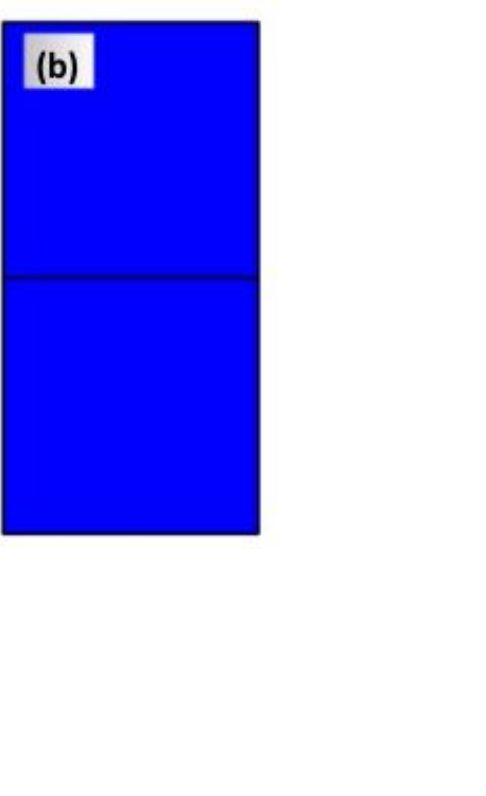

$$
\text { . }
$$
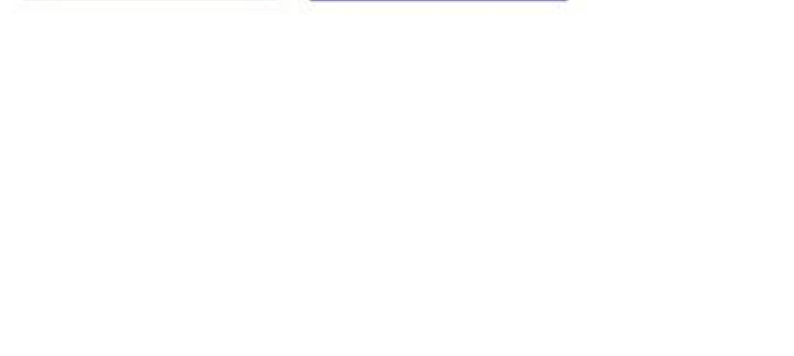